\title{
Erupção pigmentar fixa à nimesulida comprovada por teste de contato
}

\author{
Fixed pigmented erythema from nimesulide proven by patch testing \\ Juliana Leocádio Martins ${ }^{1}$, Tânia Maria Gonçalves Gomes ${ }^{1}$, José Luiz Magalhães Rios ${ }^{1,2}$, \\ Marilúcia Alves Venda ${ }^{1}$, Kleiser Aparecida Mendes ${ }^{1}$
}

\section{RESUMO}

A erupção pigmentar fixa (EPF) é uma reação cutânea adversa a drogas relativamente comum, envolvendo cerca de $10 \%$ de todas as reações de hipersensibilidade a medicamentos (RHM). Envolve uma reação imunológica não imediata, mediada por células T CD8+ sensibilizadas, relacionada ao mecanismo do tipo IVc na classificação de Gell e Coombs. Um dos grupos mais frequentemente implicados nesse tipo de reação é o dos antiinflamatórios. Relatamos o caso de um homem que, 24 horas após iniciar tratamento com nimesulida para lombalgia, apresentou um quadro de lesões cutâneas tipo máculas eritemato-violáceas bem delimitadas e disseminadas pelo corpo. A nimesulida é um fármaco anti-inflamatório não esteroidal (AINE) pertencente à classe das sulfonanilidas, que atua como inibidor seletivo da enzima da síntese de prostaglandina, a ciclo-oxigenase, inibindo preferencialmente a COX-2. O diagnóstico foi comprovado pela realização do teste de contato, também conhecido como patch test, que traduziu positividade na segunda leitura realizada após 72 horas da sua colocação.

Descritores: Erupção por droga, anti-inflamatórios não esteroidais, testes do emplastro, hipersensibilidade a drogas.

\section{Introdução}

A erupção pigmentar fixa (EPF) é uma reação cutânea adversa a medicamento relativamente comum, envolvendo cerca de $10 \%$ das reações de hipersensibilidade a drogas, ocorrendo em qualquer idade, sendo mais comum em adultos jovens ${ }^{1,2}$.

\section{ABSTRACT}

Fixed pigmented erythema (FPE) is a relatively common adverse drug reaction, consisting of approximately $10 \%$ of all drug hypersensitivity reactions. It involves a non-immediate immune reaction mediated by sensitized $C D 8+T$ cells and related to the type IVc mechanism in the Gell-Coombs classification. One of the groups most frequently involved in this type of reaction is that of anti-inflammatory drugs. We report the case of a man who, 24 hours after starting treatment with nimesulide for low back pain, presented with well-defined cutaneous lesions consisting of erythematous-violaceous macules and spread throughout the body. Nimesulide is a non-steroidal anti-inflammatory drug (NSAID) belonging to the sulfonanilide class that acts as a selective inhibitor of the prostaglandin synthesis enzyme, cyclooxygenase (COX), preferentially inhibiting COX-2. The diagnosis was confirmed by a patch test, which translated positively in the second reading performed 72 hours after its placement.

Keywords: Drug eruptions, non-steroidal anti-inflammatory drugs, patch testing, drug hypersensitivity.

Relaciona-se a uma resposta alérgica do tipo tardia, mediada por células T citotóxicas CD8+, com reativação das células $T$ residentes de memória na epiderme e derme a cada nova exposição $0^{3,4}$. A história clínica e as características marcantes das

1. Hospital Central do Exército, Alergologia e Imunologia - Rio de Janeiro, RJ, Brasil.

2. Faculdade de Medicina de Petrópolis, Alergologia e Imunologia - Petrópolis, RJ, Brasil. 
lesões (eritemato-violáceas, arredondadas em pele e mucosas, que recorrem no mesmo lugar após a reexposição, deixando uma hipercromia residual até o seu desaparecimento total, provocadas pelo uso da droga) sugerem o diagnóstico que pode ser identificado e confirmado pelos testes de provocação oral (TPO) e/ou contato com a droga suspeita ${ }^{5,6}$.

A nimesulida é um medicamento muito usado na atualidade para tratamento de diversas patologias por conta do seu efeito anti-inflamatório, antipirético e analgésico, sendo, portanto, um elemento relevante apontado como fator causal suspeito, assim como alguns antibióticos, analgésicos, anti-hipertensivos, anticonvulsivantes, dentre outros ${ }^{7,8}$.

O TPO é padrão ouro para o diagnóstico da EPF, mas não é isento de risco, como eventualmente acontece, evoluindo para a forma grave com bolhas disseminadas, denominada de erupção pigmentar fixa bolhosa generalizada ${ }^{9}$. $O$ teste de contato com a medicação suspeita é seguro, está indicado nas reações de hipersensibilidade imunológica não imediata e deve ser realizado entre seis semanas e seis meses após a resolução do quadro cutâneo ${ }^{10-12}$.

Somente algumas medicações têm concentrações padronizadas para o teste de contato ${ }^{13-15}$. A preparação extemporânea é uma forma de testar medicações não padronizadas ${ }^{16}$.

O tratamento é principalmente a identificação e suspensão da medicação responsável pelo quadro cutâneo, podendo ser necessário o uso de corticoide tópico ou oral para controle da reação ${ }^{17}$.

\section{Descrição do caso}

Paciente masculino de 55 anos referia que sete dias antes da consulta apresentara lombalgia e procurou atendimento no setor de emergência, tendo sido prescrito Dipirona ${ }^{\circledR} 1 \mathrm{~g}$ (dipirona), Maxsulid ${ }^{\circledR}$ 400 mg (nimesulida), Miosan ${ }^{\circledR} 5$ mg (ciclobenzaprina) e Tylex ${ }^{\circledR} 30$ mg (paracetamol + codeína) para alívio da dor. Após vinte quatro horas do início do tratamento, o paciente apresentou lesões cutâneas eritematovioláceas, arredondadas, que não desapareciam à digitopressão, distribuídas pelos membros inferiores, região palmar e primeiro quirodáctilo esquerdos, lábios (associada a discreto edema) e palato. Estas lesões eram ora pruriginosas ora dolorosas ao exame e evoluíram para máculas acastanhadas.

O paciente retornou ao setor de emergência três dias após o início do quadro cutâneo, onde foi medi- cado com corticoide e prometazina injetáveis com meIhora parcial do prurido e da dor, porém com a persistência das lesões. Foi, então, encaminhado ao Serviço de Alergia para avaliação e acompanhamento.

Na primeira consulta, relata reação semelhante, nos mesmos locais, cerca de dois meses antes, após uso de ibuprofeno e outras medicações que não recordava os nomes para um tratamento dentário. Nessa ocasião, suspendeu a medicação assim que a reação cutânea se iniciou e as lesões evoluíram para uma hipercromia residual. Relata, ainda, ter usado Dorflex ${ }^{\circledR}$ (dipirona + orfenadrina + cafeína) no intervalo entre as crises, sem quaisquer intercorrências.

No momento da consulta, o paciente apresentavase em bom estado geral, sem quaisquer outras alterações que não as máculas eritemato-violáceas distribuídas nos membros inferiores, mão esquerda e palato e outras acastanhadas nos pés (Figura 1).

Devido à hipótese diagnóstica de erupção pigmentar fixa, o paciente foi orientado a suspender medicamentos que continham ibuprofeno e nimesulida, utilizar paracetamol nos casos de dor, e agendar teste de contato com nimesulida e ibuprofeno.

O teste de contato foi realizado seis semanas após a resolução do quadro clínico. Foram testados a nimesulida e o ibuprofeno nas concentrações de $10 \%$ do princípio ativo em vaselina (na forma manipulada), e $30 \%$ da forma comercial (comprimido macerado), em vaselina sólida como preparação extemporânea, além de vaselina como controle negativo. As preparações foram aplicadas no dorso (área sem lesão) e na face posterior da coxa esquerda (área de lesão residual). As leituras foram realizadas com 48 e 72 horas após a colocação do teste, sendo positiva (+) à nimesulida na segunda leitura, em ambas as concentrações, na região da coxa. Ibuprofeno e controle negativo não apresentaram reatividade (Figura 2 ).

\section{Discussão}

As reações de hipersensibilidade a fármacos afetam cerca de $7 \%$ da população em geral, sendo os anti-inflamatórios não esteroides (AINEs) responsáveis por $21-25 \%$ dessas reações ${ }^{18}$. É de fundamental importância a identificação do agente causal a fim de evitar novas exposições e, consequentemente, novas crises.

No caso relatado, a positividade para a nimesulida define o responsável pela reação, comprovando assim 

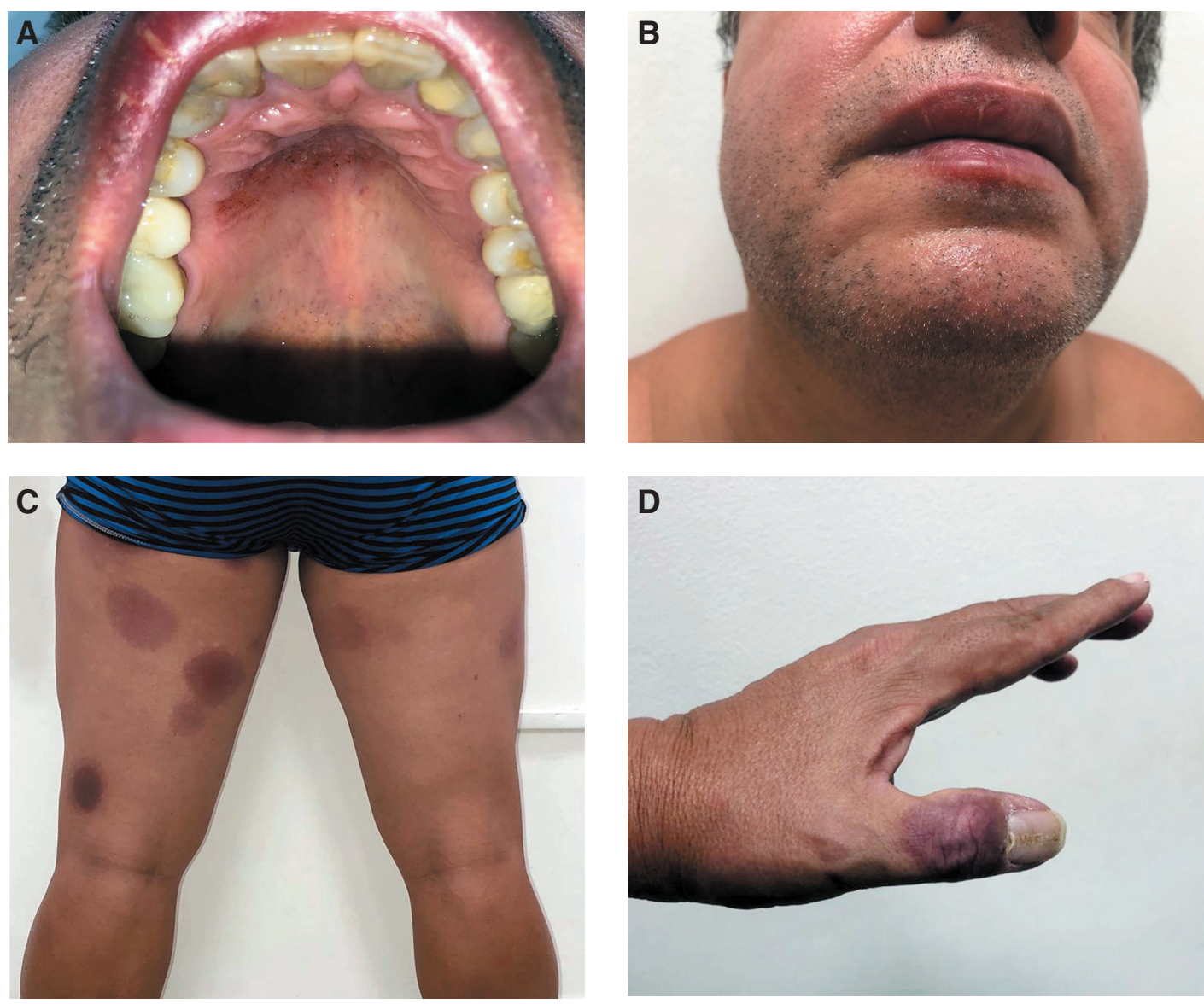

Figura 1

Máculas eritemato-violáceas: $(A)$ palato, $(B)$ lábio, $(C)$ face posterior da coxa e (E) mão
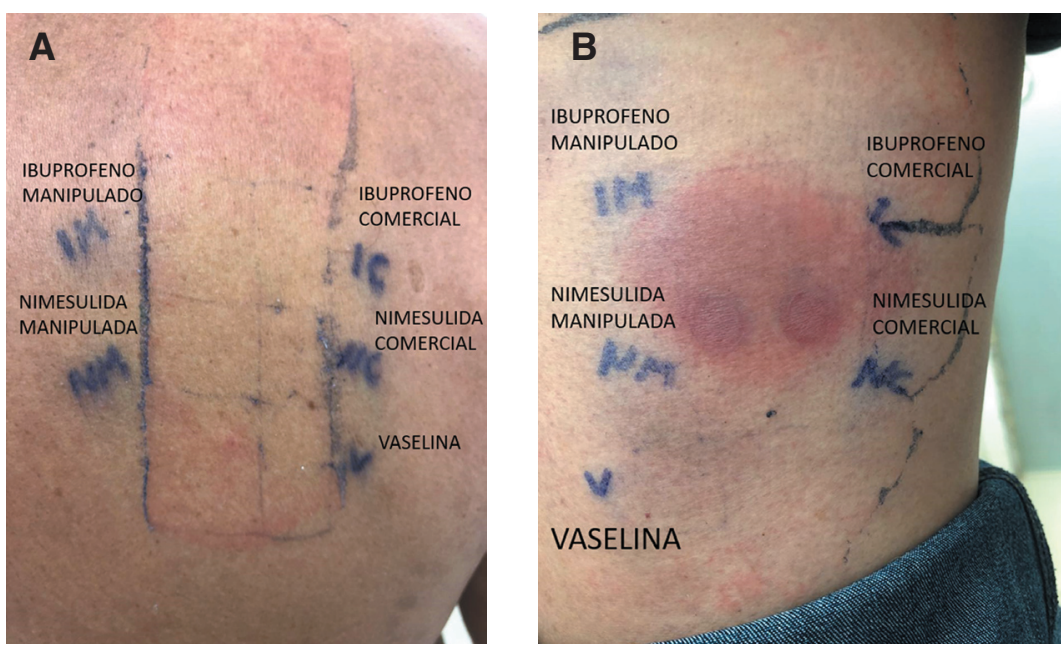

Figura 2

Teste de contato: (A) leitura de 72 horas no dorso (pele sem lesão), (B) leitura de 72 horas (lesão residual) 
o diagnóstico de EPF. Nos pacientes com EPF, as substâncias devem ser aplicadas na pele sem lesão (área sã, preferencialmente no dorso) e no local de lesão residual. O resultado, como no teste de contato padrão, segue os critérios do International Contact Dermatitis Research Group (negativo, duvidoso, positivo,+++ ou +++ ), após as leituras realizadas em 48 e 72 horas.

Mesmo que as concentrações quanto à padronização e validação ainda não estejam disponíveis para todos os fármacos, o teste de contato é uma ferramenta de fácil execução, segura e eficaz. Através deste, demonstrou-se, com bastante relevância clínica, a reprodutibilidade considerável da lesão, confirmando a farmacodermia em questão, além de ter possibilitado identificar outros fármacos alternativos seguros para o uso.

\section{Referências}

1. Patel S, John AM, Handler MZ, Schwartz RA. Fixed Drug Eruptions: An Update, Emphasizing the Potentially Lethal Generalized Bullous Fixed Drug Eruption. Am J Clin Dermatol. 2020 Jun;21(3):393-9.

2. Malheiro D, Cadinha S, Rodrigues J, Vaz M, Castel-Branco MG. Nimesulide-induced fixed drug eruption. Allergol Immunopathol (Madr). 2005;33(5):285-7.

3. Quint T, Wöhrl S, Kinaciyan T. Fixed drug eruption caused by fluconazole - an underdiagnosed but recurrent problem. Contact Dermatitis. 2019;80(3):172-3.

4. Flowers $\mathrm{H}$, Brodell $\mathrm{R}$, Brents M. Fixed drug eruptions: presentation, diagnosis, and management. South Med J. 2014;107(11):724-7.

5. Sehgal VN, Srivastavaet G. Fixed drug eruption (FDE): changing scenario of incriminating drugs. International Journal of Dermatology. 2006;45(8):897-908

6. Zedlitz S, Linzbach L, Kaufmann R, Boehncke W. Reproducible identification of the causative drug of a fixed drug eruption by oral provocation and lesional patch testing. Contact Dermatitis. 2002;46(6):352-3.

7. Butler DF, Vinson RP, Callen JP. Fixed drug eruptions. Medscape [site da Internet]. Disponível em:https://emedicine.medscape.com/ article/1336702-workup\#c5.
8. Mahboob A, Haroon TS. Drugs causing fixed eruptions: a study of 450 cases. Int JDermatol. 1998;37(1):833-8.

9. Mitre V, Applebaum DS, Albahrani Y, Hsu S. Generalized bullous fixed drug eruption imitating toxic epidermal necrolysis: a case report and literature review. Dermatol Online J. 2017;23(7):1-4. http://dx.doi.org/10.5070/D3237035734.

10. Motta AA, Kalil J, Barros MT. Testes cutâneos. Rev Bras Alerg Imunopatol. 2005;28(3)73-83.

11. Aun MV, Cocco RR, Mello Y. Testes in vivo nas reações de hipersensibilidade a medicamentos. Arq Asma Alerg Imunol. 2018;2(4):390-8

12. Zinn Z, Gayam S, Chelliah MP, Honari G, Teng J. Patch testing for nonimmediate cutaneous adverse drug reactions. J Am Acad Dermat. 2018;78(2):421-3.

13. Barbaud A. Skin testing and patch testing in non-IgE-mediated drug allergy. Curr Allergy Asthma Rep. 2014:14(6):442.

14. Barbaud A, Collet E, Milpied B, Assier H, StaumontD, Avenel-Audran $\mathrm{M}$, et al. A multicentre study to determine the value and safety of drug patch tests for the three main classes of severe cutaneous adverse drug reactions. Br J Dermatol. 2013;168(3):555-62.

15. Johansen JD, Aalto-Korte K, Agner T, Andersen KE, Bircher A, Bruze $\mathrm{M}$, et al. European Society of Contact Dermatitis guideline for diagnostic patch testing - recommendations on best practice. Contact Dermatitis. 2015;73(4):195-221.

16. Assier H, Valeyrie-Allanore L, Gener G, Verlinde Carvalho M, Chosidow $\mathrm{O}$, Wolkenstein P. Patch tests in cutaneous adverse drug reactions; value of extemporaneous patch tests. Contact Dermatitis. 2017:77(5);297-302.

17. Sehgal VN, Gangwani OP. Fixed drug eruption: current concepts. Int J Dermatol. 1987;26(2):67-74.

18. Kowalski ML, Makowska JS, Blanca M, Bavbek S, Bochenek $\mathrm{G}$, BousquetJ, et at. Hypersensivity reactions to nonsteroidal anti-inflamatory drugs (NSAIDs) - classification, diagnosis, and management: review of the EAACI/ENDA and GA2LEN/HANNA. Allergy. 2011;66:818-29.

Não foram declarados conflitos de interesse associados à publicação deste artigo.

Correspondência:

Juliana Leocádio Martins

E-mail: julianaleocadiomartins@gmail.com 